\title{
Generation-IV nuclear reactor systems
}

\author{
R. Caciuffo \\ European Commission, Joint Research Centre \\ Postfach 2340, DE-76125 Karlsruhe, Germany \\ C. FAZIO \\ European Commission, Joint Research Centre \\ Westerduinweg 3, NL-1755 LE Petten, The Netherlands \\ C. Guet \\ Energy Research Institute@NTU, Nanyang Technological University \\ 1 CleanTech Loop, 06-04, 637141 Singapore
}

\begin{abstract}
Summary. - In this paper, we provide a concise description of the six nuclear reactor concepts that are under development in the framework of the Generation-IV International Forum. After a brief introduction on the world energy needs, its plausible evolution during the next fifty years, and the constraints imposed by the necessity to address the climate challenges we are facing today, we will present the main features of the innovative nuclear energy systems that hold the promise to produce almost-zero-carbon-emission electricity, heat for chemistry and industrial manufacturing, hydrogen to be used as energy vector, and affordable freshwater. Potential advantages over currently available nuclear systems in terms of increased safety, reduced proliferation risks, economical affordability, sustainability of the fuel cycle, and management of the waste inventory will be critically discussed against the technical challenges that remain to be overcome.
\end{abstract}

\section{1. - Powering the world while ensuring climatic stability}

The energy need of the World continues to grow at a fast pace. According to the Global Energy and $\mathrm{CO}_{2}$ Status Report 2018 of the International Energy Agency [1], the energy demand rose by $2.3 \%$ in 2018 , bringing the energy-related emissions of $\mathrm{CO}_{2}$ 
to $\sim 33$ gigatonnes. With a population of about 7.6 billion, the electricity demand is approaching $24 \mathrm{PWh}$ /year, about $20 \%$ of the total final energy consumption. At the same time, about one billion people have still no access to electricity. This is expected to change and, while the population is expected to reach 11.2 billion by the end of the 21st century [2], the electricity demand should more than double. As greenhouse gas (GHG) emissions are threatening the climatic stability and make dramatic dislocations in economic and social structures impending, immediate action is required to change energy production and consumption models.

Improvements in the consumption efficiency, novel paradigms in the transport sector, and electricity generation hold the largest potential for the reduction of $\mathrm{CO}_{2}$ emission, which makes up to $\sim 3 / 4$ of the anthropogenic greenhouse effect. The amount of GHG emissions is, of course, strictly related to the primary energy mix. For instance, whereas the combustion of lignite or coal results in about 1 tonne of $\mathrm{CO}_{2}$ per $\mathrm{MWh}$ of produced energy, burning gas reduces the emission by a factor $\sim 2$, photovoltaic by a factor $\sim 10$, nuclear by a factor 50-100, wind and hydro-power by a factor $\sim 100$ [3]. In 2017, most of the gross inland consumption in the European Union $(\sim 74.1 \%)$ came from the combustion of fossil fuels. Solid fuels provided $\sim 14.5 \%$, petroleum $\sim 36.4 \%$, and natural gas $\sim 23.2 \%$ of the total energy available. Nuclear heat and renewable energy products (such as hydro, wind and solar energy) contributed a share of about $12.3 \%$ and $13.6 \%$, respectively [4] (it must be noticed that the almost equal share of nuclear and renewable energy production requires an installed renewable capacity much higher than the nuclear one).

Figure 1 shows the instantaneous emission of $\mathrm{CO}_{2}$ in different countries of the European Union on a early autumn day of 2019 [5], together with the amount of nuclear power generated in each of them [6]. A correlation between low $\mathrm{CO}_{2}$ emission and high nuclear share in the energy mix is evident. Norway, where the lowest emission is recorded, is an exception because of the dominant contribution of hydropower to electricity production. Carbon capture and storage technologies could mitigate the impact of fossil fuels on the composition of the atmosphere. However, many problems remain to be solved before these technologies become applicable at large scale, while nuclear energy is immediately employable and could provide an efficient solution for achieving deep decarbonisation goals at short term.

1'1. The current nuclear reactors fleet and its share in the energy mix. - Thirty countries in the world exploit nuclear power plants for electricity generation. The 454 operational installations have a capacity of $400 \mathrm{GW}_{e}, \sim 14 \%$ of the global electricity production (in the following, we will indicate with $\mathrm{W}_{e}$ the unit of power of produced electricity). In 2018, the installed nuclear power in the European Union was $118 \mathrm{GW}_{e}$ [6]. The 166 nuclear power plants operating in 14 of the Union's Member States covered $\sim 25 \%$ of the electricity needs. Fifty-five new reactors are currently under construction (16 in Europe), mainly in South Asia and the Far East. In many countries, however, the deployment of nuclear power is hindered by a lack of public acceptance. A survey conducted by Ipsos MORI after the Fukushima accident showed that a vast majority of 


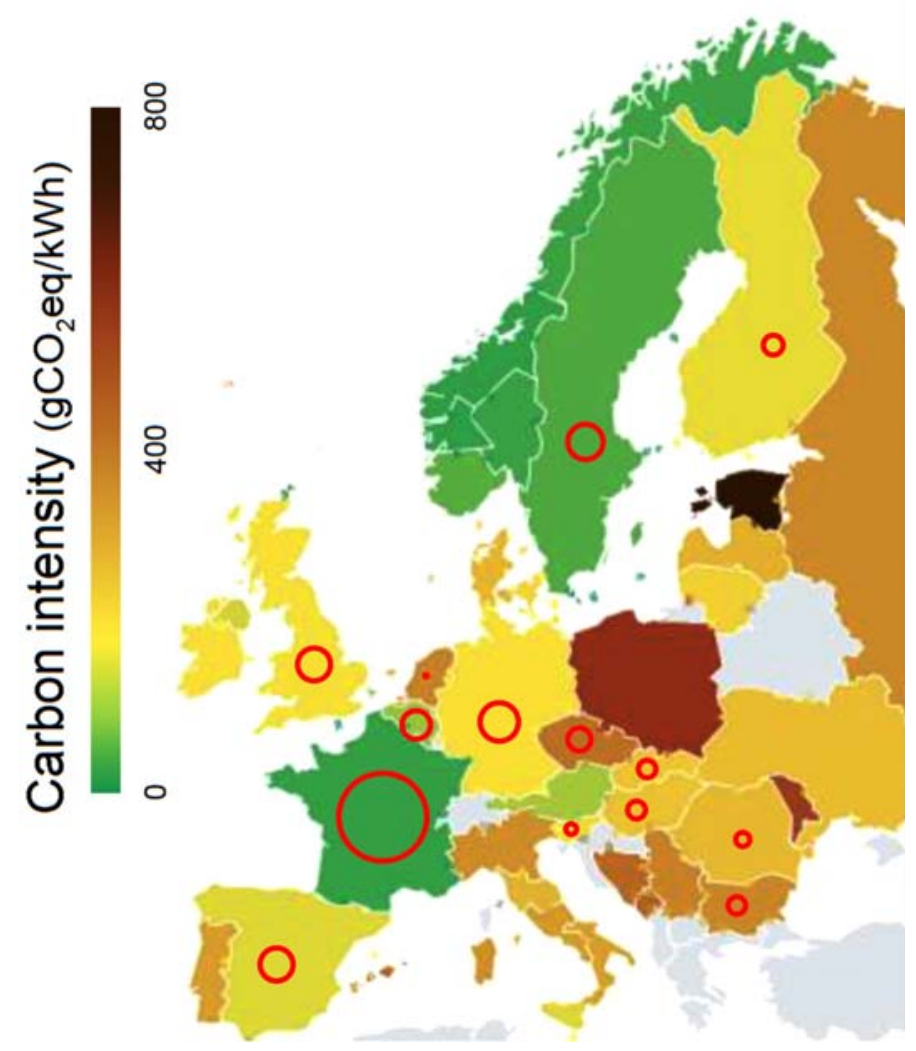

Fig. 1. - Carbon intensity of electricity generation in the European Union countries provided by the electricityMap application (available at https://www.electricitymap.org) at 10:35 a.m. on 25 September 2019. The area of the circles is proportional to the heat produced by nuclear power plants in the 14 Member States where the nuclear option is part of the energy mix (going from $\sim 1.2 \mathrm{PWh} /$ year in France to $\sim 9.2 \mathrm{TWh} /$ year in the Netherlands).

the public oppose nuclear energy as a mean of electricity production (with a world average of $62 \%$ of opponents and a peak of about $80 \%$ in Italy, Germany, and Mexico; a favourable opinion was reported only for India, Poland, and the USA with $39 \%, 43 \%$, and $48 \%$ of opponents, respectively). Despite the outstanding safety performance demonstrated so far, with an estimated core damage frequency of $\sim 10^{-6} /$ year, nuclear remains a controversial addition to low-carbon energy plans.

Whilst a large number of nuclear reactor concepts were proposed and developed at the dawn of the nuclear age during the 1950s and early 1960s, about $82 \%$ of the nuclear reactors operated today are Light Water Reactors (LWR), that is systems using natural water as a neutron moderator, coolant, and heat carrier. In the most common LWR design, the Pressurized Water Reactor (PWR), water is kept at high pressure to prevent boiling and is used to transfer the heat generated in the core to a heat exchanger where the vapour is produced. On the contrary, in a Boiling Water Reactor (BWR), the cooling 
water is brought to boiling in the core and the steam is directly powering the turbines. The neutronics design is more complex than for a PWR, because of the two-phase coolant, but the absence of a secondary cooling circuit reduces the construction costs.

LWRs are commonly used for baseload electricity generation and during the past 60 years have demonstrated high reliability and excellent performance. They are, however, not ideal machines. A flexible operation is difficult, due to e.g. the build-up of fission products with a high neutron capture probability, like xenon-135, that severely affects the neutron balance preventing easy power cycling. Direct use of the heat developed in the reactor core is strongly limited by the relatively low outlet temperature ( $598 \mathrm{~K}$ for PWR and $561 \mathrm{~K}$ for BWR), which also limits the efficiency of the thermodynamic cycle for the heat-to-electricity conversion. Moreover, LWRs do not allow optimal and sustainable use of the raw material (natural uranium) used for fuel fabrication. As they work with thermal neutrons and use water as moderator, these reactors require fuel enriched to about 3-4\%. LWRs burn only the U-235 isotope and some Pu isotopes that are produced in the fuel core. Indeed the efficiency is very low as natural uranium contains only $0.7 \%$ of the fissile U-235 isotope. To a certain extent, this can be improved by increasing the ratio between the fuel and the moderator volumes. As a result of the increase of the average neutron energy, the conversion of fertile U-238 into fissile $\mathrm{Pu}$ 239 is enhanced. This allows for reactor designs with heterogeneous cores (containing driver fuel assemblies and depleted uranium targets) and the use of plutonium directly introduced in the fuel rods (mixed U-Pu oxides or MOX).

With about $10^{4}$ tons of spent nuclear fuel discharged yearly (fitting inside a cube of approximately $10 \mathrm{~m}$ side), a further challenge presented by LWRs is the management and disposal of the high-level nuclear wastes they produce. The spent nuclear fuel contains large amounts of $\mathrm{Pu}$ and minor actinides $(\mathrm{Np}, \mathrm{Am}, \mathrm{Cm}$ ) isotopes (about 12 and $3 \mathrm{~kg}$, respectively, per tonne of $\mathrm{LWR}$ spent fuel with a burnup of $50 \mathrm{GWd} / \mathrm{t}$ ) requiring safe disposal for periods of the order of $10^{5}-10^{6}$ years. A permanent solution harmonized at international level is not yet available. In several countries (e.g. U.S.A. and European Union), the official policy is deep geological disposal at depths between $250 \mathrm{~m}$ and $1000 \mathrm{~m}$ for mined repositories, or $2 \mathrm{~km}$ to $5 \mathrm{~km}$ for boreholes.

Lastly, with a typical capacity of $1 \mathrm{GW}_{e}$ LWRs are very large installations, requiring long construction times and huge capital investments. In this respect, considerations are given to Small Modular Reactors (SMR) in order to ease construction and reduce costs.

1'2. Future concepts. - To overcome the back-end of the fuel cycle limitations and to extend the fields of applications of currently available nuclear reactors, several alternatives holding the promise to power the world with almost-zero-carbon electricity are being investigated worldwide. The effort is coordinated by the Generation-IV International Forum, a framework for international co-operation that was set up to carry out research for the next generation of nuclear energy systems [7]. Six concepts have been selected, with core designs including thermal and fast neutron spectra, closed and open fuel cycles. All of them are the outcome of revisiting technologies already explored during the 1950s and 1960s. 
This new generation of nuclear reactors will be optimized for flexible electricity generation, direct heat production for industrial manufacturing and chemical processes, and other applications such as desalination of seawater and large-scale production of hydrogen by water high-temperature electrolysis or steam reforming. The latter industrial applications require very high output temperatures to power industrial furnaces used, for instance, by metallurgical and chemical industries that today are responsible for a large portion of greenhouse gas emissions. An important goal of Generation-IV reactors is to increase safety, for instance by allowing one to use the passive physics of the reactor system to shut the reactor down and remove residual heat in off-normal conditions. An ideal system should be resilient against proliferation risks (misusing nuclear reactors to gain fissile materials for nuclear weapons fabrication), producing small amounts of easily separable plutonium-239 or uranium-233, or even allowing the irradiation of plutonium available from military stockpiles. Furthermore, it should make the use of natural uranium more sustainable by allowing higher burnups (the amount of energy produced per mass of used fuel; $100-200 \mathrm{GWd} /$ tonne can be achieved in GenIV reactors, compared to 50-100 GWd/tonne for LWRs) and by allowing breeding, that is by enhancing the production of fissile isotopes (U-233 and $\mathrm{Pu}-239$ ) by conversion of fertile materials (Th-232 and U-238, respectively; in the case of U-238, fast neutrons are needed for breeding; see next section). The burdens of waste management, both in terms of space and timespan, should be alleviated by the possibility to burn plutonium and long-living minor actinides $(\mathrm{Np}, \mathrm{Am}, \mathrm{Cm})$ contained in LWRs spent fuel and by a reduced overall production of high-level waste. The former goal would be achievable in fast neutron reactors and, primarily, in the specifically designed accelerator-driven subcritical systems, like MYRRHA that is now under construction in Belgium, as described in the contribution of Hamid Ait Abderrahim in these proceedings. The advantage of separation and transmutation (cyclical chemical processing of nuclear fuel to harness $\mathrm{Pu}$ and minor actinides to be further irradiated and transmuted into short-living radionuclides) in terms of shortening the required sequestration time (from the biosphere) is shown in fig. 2, where the time evolution of the ingestion radiotoxicity of one tonne of the typical used nuclear fuel is compared with that of the same fuel submitted to partitioning and transmutation cycles. This evolution of radiotoxicity is a relevant aspect to assess intentional or unintentional human intrusion scenarios in geological repositories.

Whilst today's nuclear power plants are very big installations, the size of future reactors will range from very small to very large. Small-modular-reactors and micro-reactors are designed to generate between $1 \mathrm{~kW}$ and $200 \mathrm{MW}$ of thermal power; reactors could be built in a factory and then shipped to remote and desert locations (for instance to the arctic or Mars) where they would produce power for decades without requiring intervention. The NASA Kilopower reactor is an example of these attempts [8]. Small modular reactors (not treated in this lecture), featuring modular construction, size scaling, fuel cycles extended up to several decades, passive safety systems, and reduced costs are conceived for making the nuclear option more attractive. 


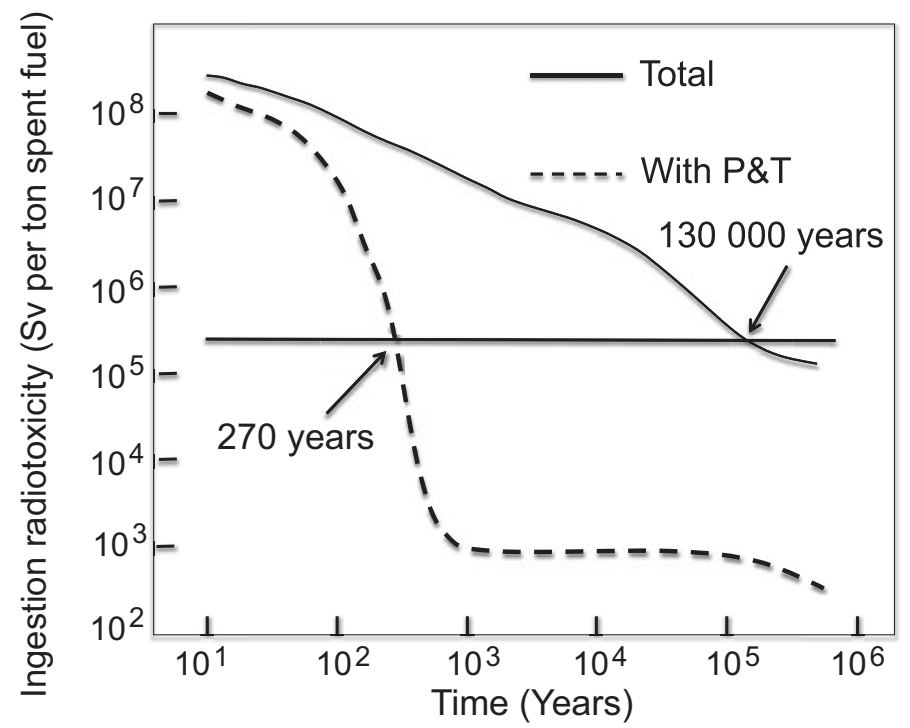

Fig. 2. - Ingestion radiotoxicity of one tonne of used nuclear fuel as a function of time, with (broken line) and without (solid line) partitioning and transmutation. The horizontal line indicates the radiotoxicity of the pristine natural material used for the fabrication of the fuel.

\section{2. - Generation-IV nuclear reactors}

2'1. Sodium-Cooled Fast Reactor (SFR). - The first nuclear reactor used to generate home electricity was a fast-neutron reactor cooled by eutectic sodium and potassium alloy, namely the Experimental Breeder Reactor 1 (EBR-1) that on December 20, 1951, lit four 200 watt electric bulbs in Butte County, Idaho [9]. EBR-1 had been designed and built to demonstrate, as Enrico Fermi had suggested, that a nuclear reactor was able to produce more fissile nuclides than fissioned. This breeding concept is based on the conversion of non-fissile material (U-238) into fissile one (Pu-239) by neutron capture and two successive beta-decays. If the conversion factor (the ratio between the number of fissile nuclides created and the number of those consumed) is larger than one, the reactor is called a breeder. Instead of "burning" only U-235 $(\sim 0.7 \%$ of natural uranium), a breeder reactor makes it possible to use also a large percentage of U-238 ( $\sim 99.3 \%$ of natural uranium) in a U-Pu cycle. With uranium fuel, breeding conditions can only be achieved with fast neutrons that provide the needed balance between the fission probability, the number of neutrons released, and the neutron loss and capture probabilities. It is, therefore, necessary to avoid the presence of light-atom materials in the core, like water or graphite, which would act as moderators and slow down neutrons, as it occurs in thermal-neutron reactors. Hence, to ensure a predominance of fast and epithermal neutrons over thermal ones in the energy spectrum, it is necessary to use a heavy-atom liquid metal as a coolant medium. 
Liquid sodium was considered a good choice as a heat transfer agent because of its favourable thermophysical and neutronics properties. An ideal fast nuclear reactor coolant should minimize neutron moderation, combine high thermal conductivity and boiling point with low melting temperature and vapour pressure. Moreover, the material should be stable under irradiation, chemically compatible with fuel and structural materials, and have a small neutron absorption cross-section. It should not become heavily activated under neutron irradiation, should not be too corrosive, and its circulation across the core should be guaranteed by a reasonably small pumping power. Sodium metal, with a melting point of $371 \mathrm{~K}$, a boiling point of $1156 \mathrm{~K}$, thermal conductivity of $89.4 \mathrm{~W} /(\mathrm{m} \mathrm{K})$ (at $371 \mathrm{~K})$, and dynamic viscosity of $6.88 \times 10^{4} \mathrm{Pas}$ (at $371 \mathrm{~K}$ ) satisfies most of these requirements [10].

As the core outlet temperature in SFR is higher than $800 \mathrm{~K}$, a thermodynamic efficiency $\eta \sim 40 \%$ can be achieved ( $\eta \sim 30 \%$ for LWRs). The power of SFR ranges between 400 to $1300 \mathrm{MW}$, with a core power density that can be as high as $300 \mathrm{MW} / \mathrm{m}^{3}$, a factor 5 higher than for a typical LWR. Liquid sodium is indeed considerably more effective than water as a heat transfer medium, so that compact cores with closely packed fuel pins (a neutronics requirement in fast reactors) can be used. As the neutron capture cross-section becomes smaller with increasing neutron speed, the reactivity loss due to the accumulation of fission products (such as xenon) is much less severe than in a thermal reactor. On the other hand, the neutrons' mean free path is much longer, making core heterogeneity effects less important. The fraction of neutrons escaping is high, and this is usually remedied by inserting a fertile blanket, that is a peripheral shell of fertile material.

With liquid sodium, cooling is performed at ambient pressure and the fluid circulation can be maintained by electromagnetic pumps, thanks to the high electrical conductivity of sodium. Large temperature gradients favour natural convection and allow relying on passive safety for residual heat removal. The low pressure operation also allows a containment vessel (usually a double-walled austenitic stainless-steel tank) much lighter than for traditional LWRs. The main safety problems with the use of liquid sodium stem from its high chemical reactivity with both water and air, with associated risks of fire or explosions, and from its tendency to ablate concrete.

While the uranium fuel for a thermal nuclear reactor typically contains less than $5 \%$ of U-235, higher initial enrichment (in U-235 or Pu-239) is needed to achieve criticality in fast reactors (because reaction cross sections are lower for fast neutrons). Fuel for SFRs can be a metal, such as a binary (U-Zr) or a ternary (U-Pu-Zr) alloy, or a ceramic mixed oxide $(\mathrm{U}, \mathrm{Pu}) \mathrm{O}_{2}$. It can also contain transuranic wastes to be transmuted by the copious available amount of neutrons with energy larger than their fission threshold. High-energy neutrons will also split U-238, increasing the neutron population and reducing at the same time the production of minor actinides. It must be noted that the amount of minor actinides that can be destroyed at each cycle is limited by severe neutronics constraints (delayed neutrons, feedback coefficient, void reactivity coefficient). The concept of partitioning and transmutation of long-lived radionuclides with fast reactors, therefore, requires repeated fuel reprocessing. Because of the associated proliferation risks (i.e. the 


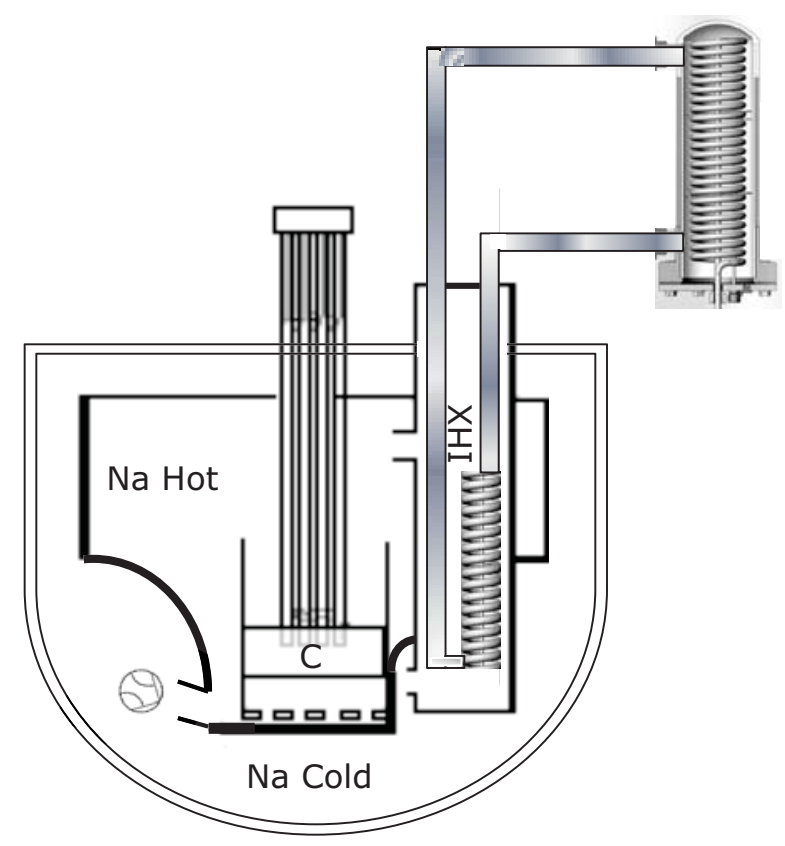

Fig. 3. - Schematic representation of a sodium-cooled fast reactor with pool configuration. C is the core, IHX is the intermediate heat exchanger.

possible diversion during reprocessing of fissile materials for weapon construction) this practice is forbidden in the U.S.A. Alternative concepts as the Accelerator Driven Transmutation Systems (see the contribution of Hamid Ait Abderrahim in these proceedings) are developed with the objective to increase the transmutation rate through the use of uranium-free fuel loaded with higher amount of minor actinides.

Two main configurations have been considered for SFRs, one in which the primary coolant leaves the reactor vessel and enter an intermediate heat exchanger outside the vessel that transfers heat from the primary loop to the steam generator (loop reactors), the other in which the core is immersed in a pool of liquid coolant containing also the intermediate heat exchanger. The latter is called a pool configuration and is the choice made in the framework of the Generation-IV initiative (fig. 3). The intermediate heat exchanger is required to avoid the possibility of contact between activated sodium and water in accidental conditions.

So far, twenty-one SFR have been operational worldwide. Six are currently working, two in Russia for electricity generation (the BN-660 and BN-800 reactors, with 600 and $800 \mathrm{MW}_{e}$ ) and four for carrying out experiments (in Japan, Russia, India, and China). Two more SFRs are under construction. The $150 \mathrm{MW}$ multipurpose sodium-cooled fast neutron research reactor (MBIR) is expected to become critical in 2024 at the site of the Research Institute of Atomic Reactors (NIIAR) at Dimitrovgrad, Russia. It will be 
a multi-loop reactor running on MOX fuel to be used for multipurpose testing including lead, lead-bismuth and gas coolants. In India, at Kalpakkam, the $1250 \mathrm{MW}\left(500 \mathrm{MW}_{e}\right)$ PFBR pool-type reactor is expected to produce electricity in 2022. It will burn MOX fuel with depleted and natural uranium and $25 \% \mathrm{Pu}$ oxide. Furthermore, several SFRs are under design.

In France, ASTRID - the Advanced Sodium Technological Reactor for Industrial Demonstration - originally planned to be a $600 \mathrm{MW}_{e}$ pool-type has been first delayed and eventually put on hold at the end of 2019. In the US, Advanced Reactor Concepts is developing the LLC ARC-100, a $100 \mathrm{MW}_{e}$ reactor with modular components and a 20-year refuelling cycle. General Electric Hitachi Nuclear Energy has designed a $425 \mathrm{MW}$ system with multiple power modules co-located underground with a spent fuel reprocessing facility. A larger capacity reactor $\left(550 \mathrm{MW}_{e}\right)$, the pool-type metal fuelled Travelling Wave Reactor (TWR), conceived to operate without refuelling, has been designed by TerraPower. In Korea, KAERI is developing the Prototype Gen-IV Sodium-cooled Fast Reactor (PGSFR), a pool-type reactor that will burn a U-Transuranium-Zr metal fuel and is supposed to become critical after 2028 with a power of $150 \mathrm{MW}_{e}$. High-power SFRs are under development in Japan (JSFR, 3530 MW, MOX Fuel), Russia (BN 1220 MW $_{e}$ ), and China (CFR $600 \mathrm{MW}_{e}$ ). In Japan, Toshiba is studying a small modular SFR concept $\left(10 \mathrm{MW}_{e}\right)$ for electricity or heat generation in remote areas.

All these reactor concepts consider several innovations in their design that call for intensive R\&D. These innovations include, for instance, the development of new structural and fuel clad materials and transuranics bearing fuels, the design and validation of low void coefficient cores, the assessment of alternative tertiary coolants with the objectives to eliminate the intermediate heat exchanger.

2'2. Lead-Cooled Fast Reactor (LFR). - LFRs use liquid lead or lead-bismuth eutectic (LBE) alloy as a heat transfer medium. They were first commissioned during the 1960s in the USSR and produced in the 1970s for powering several Alfa-class submarines. These reactors (model OK-550 and BM-40A) were powered by highly enriched uranium and designed to run 15 years without refuelling. Earlier attempts in the U.S. of developing a natural U-Bi liquid metal fuel had been soon dismissed because of problems created by the corrosion of structural materials and because, for similar conditions, a liquid lead circuit requires higher pumping power than a liquid sodium one, due to a higher-pressure drop.

Liquid lead and lead-bismuth eutectic have a smaller thermal conductivity than sodium and moreover, to limit erosion, the coolant flow velocity in LFRs must be lower than in SFRs. This may impact the core design to ensure efficient cooling. In addition, while the irradiation-induced radioactivity of sodium (without impurities) requires about 10 years to decay, in the case of $\mathrm{Pb}$ the decay time is of the order of $10^{5}$ years. Bismuth, on the other hand, produces Po-210 under neutron irradiation (an $\alpha$ emitter with high radiotoxicity forming volatile aerosols), making tight confinement an absolute necessity. An advantage of $\mathrm{Pb}$ is the large difference between melting and boiling point $(600 \mathrm{~K}$ and $2018 \mathrm{~K}$ ), providing a large margin of safety in operating conditions (even larger for LBE alloy with a melting temperature $398 \mathrm{~K}$ ). Typical coolant outlet temperatures in LFRs 


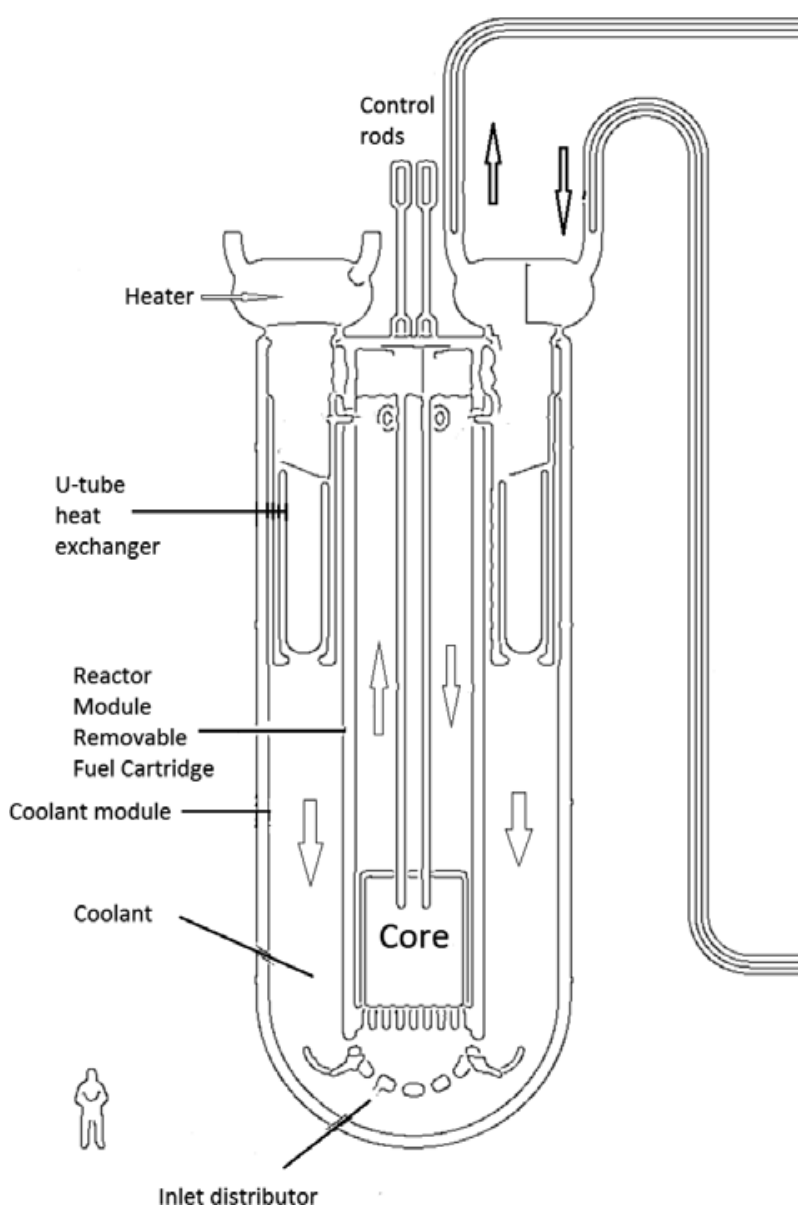

Fig. 4. - Schematic representation of a lead-cooled fast reactor with pool configuration.

range between $823 \mathrm{~K}$ and $1073 \mathrm{~K}$, allowing for direct industrial applications of the heat produced in the core.

As lead does not react disruptively with water or air, steam can be produced by putting feed water in direct contact with the coolant. The steam generator can be installed inside the reactor vessel and, as the coolant circulation can be maintained by the buoyancy of gas bubbles, primary pumps can be eliminated (fig. 4). Flow blockage due to $\mathrm{Pb}$ freezing during overcooling transients must, of course, be avoided as it would severely damage the core. Corrosion and erosion of structural materials must be carefully considered and extensive research on promising materials, such as SiC ceramics or steel lined with alumina forming alloys, continues to be carried out in combination with research on appropriate coolant chemistry control systems. It must be noted that the structural design of an LFR is a demanding task, as seismic effects are enhanced by 
the large mass of lead contained in the system ( $\sim 10^{4}$ tonnes for a medium-size plant).

LFRs can run with U-(10\%Zr) metallic fuel, or MOX, or nitrides and ensure a core life up to 15-30 years. Some designs foresee a cartridge core, to be sent back to the provider at the end of the cycle. LFR can have a typical capacity of $120-600 \mathrm{MW}_{e}$ and allow efficient use of natural resources, requiring about $1 / 3$ of uranium than LWRs, at equal power production.

In the framework of the GenIV initiative, a European consortium is developing the Advanced Lead Fast Reactor European Demonstrator (ALFRED), a $300 \mathrm{MW}\left(125 \mathrm{MW}_{e}\right)$ reactor which is planned to go critical between 2035 and 2040. A further European concept is the Swedish Advanced Lead Reactor (SEALER), designed for operation in the Arctic regions (2.4 tons of $19.9 \%$ enriched $\mathrm{UO}_{2}$ fuel, $10 \mathrm{MW}_{e}$ of power, and 30 years core life) and for connection to the UK grid (19.8 tons of $11.8 \%$ enriched UN, $55 \mathrm{MW}_{e}$, and 25 years core-life).

2:3. Gas-Cooled Fast Reactor (GFR). - The idea of using a gas instead of liquid metal for cooling a fast breeder reactor was first put forward in the 1960s, and several alternatives including helium, $\mathrm{CO}_{2}$, and steam were explored $[11,12]$. A historical overview of gas-cooled fast reactors is given in ref. [13]. General Atomics, in the U.S., conducted first design studies of a gas-cooled fast breeder reactor during the $1960 \mathrm{~s}-1970 \mathrm{~s}$ (a $300 \mathrm{MW}_{e}$ demonstration plant to be followed by a $1 \mathrm{GW}_{e}$ commercial plant). Several projects were also developed in the Soviet Union, UK, Germany, and Japan. The task, however, turned to be a very challenging and problematic one, mainly due to the difficulty to solve safety-related issues, and no prototypes have ever been constructed.

Because the number of atoms per unit volume in a gas is smaller than in a liquid, a gas coolant is not effective as a moderator even if composed by light-mass atoms. On the other hand, a gas coolant such as helium is chemically inert, making in theory intermediate coolant loops redundant, is not corrosive (when impurities as C, O, and N are kept under control), and is not activated by neutron irradiation. The hot gas leaving the core at high temperature could, in principle, be used directly in a gas turbine for electricity production. From the neutronics point of view, the use of a homogeneous coolant such as a gas presents some advantages in terms of stability of the reactivity (the formation of bubbles in a liquid coolant affects the multiplication factor $k_{e f f}$, i.e. the average number of new fissions due to the neutrons produced by an initial fission; depending on the system, this so-called void coefficient can produce either an increase or a decrease of reactivity). Moreover, the low number density, minimising parasitic effects, allows increasing the distance between fuel elements (open-packed core), which favour breeding of fertile nuclides in peripheral blankets.

On the other hand, although the specific heat of helium is high, its low density implies the need for a high-pressure system $(\geq 7 \mathrm{MPa})$, imposing safety precautions for depressurization, as for instance in a loss of coolant event, whereas the low thermal conductivity requires high coolant velocity $(\sim 100 \mathrm{~m} / \mathrm{s})$. This implies higher pumping power and less effective heat transfer than with liquid metal coolants.

Several GFR concepts are under consideration in the framework of the Generation- 


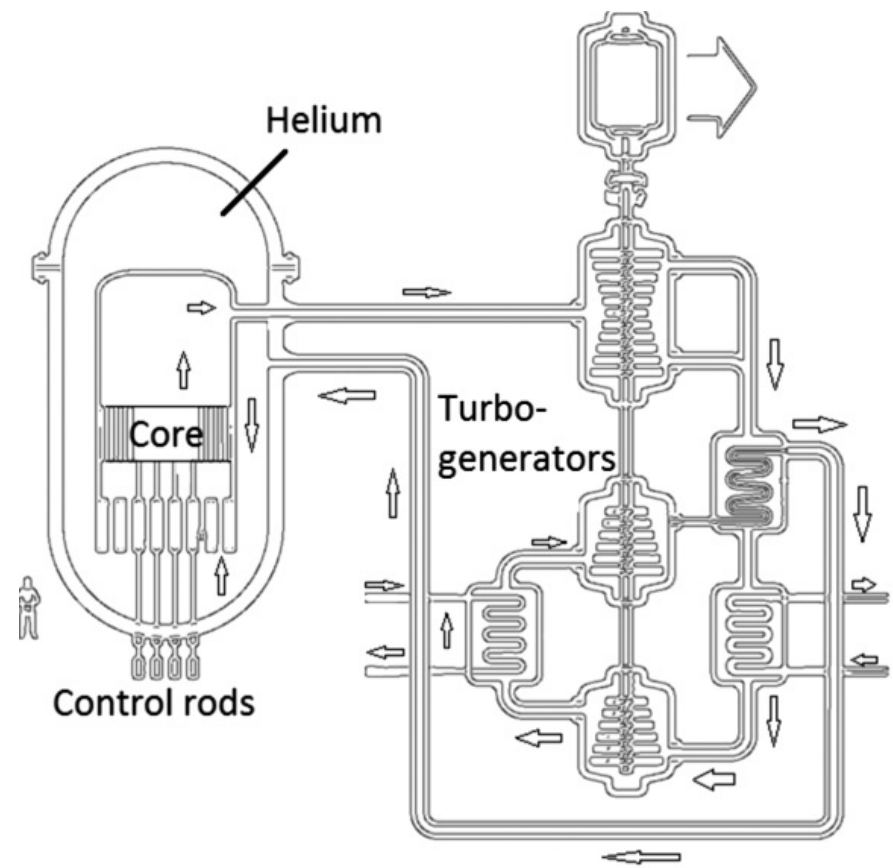

Fig. 5. - Schematic representation of a gas-cooled fast reactor with indirect cycle loops consisting of an heat exchanger and an integrated high-speed turbogenerator.

IV initiative, mainly with a focus on the optimal use of natural resources, see ref. [14] and references therein. An initial goal of developing a unit power of $600 \mathrm{MW}$ with a gas turbine for direct energy conversion was abandoned in 2006, due to considerable technical difficulties. The present focus is on a $2400 \mathrm{MW}$ power plant using three indirect cycle loops, each consisting of a heat exchanger and an integrated asynchronous high-speed turbogenerator (fig. 5). High back-up pressures must be guaranteed for several hours after a depressurization event to allow passive decay heat removal, implying a small containment building. The heat exchangers are located well above the core to have a sufficient flow rate by natural circulation (not shown in fig. 5).

An outlet coolant temperature of $1123 \mathrm{~K}$ and operating pressure of $7 \mathrm{MPa}$ will result in high thermodynamic efficiency $(\eta \sim 48 \%$ ). The high operating temperatures, however, require the use of a refractory structural material (ceramics such as $\mathrm{SiC}, \mathrm{Zr}_{3} \mathrm{Si}_{2}, \mathrm{TiN}$ ), because stainless steel would be inadequate, implying an important R\&D program to qualify these materials for nuclear use.

For proliferation resistance considerations, a fertile blanket is not foreseen. The reactor should breed just enough fissile material for refuelling in a closed fuel cycle scenario, requiring only the addition of U-238 (zero-breeding gain). This fixes the U-Pu fuel composition to $80-85 \%$ U-238 and imposes geometrical constraints (namely on the height to diameter ratio of the core) that must be compromised with those imposed by thermofluidodynamics requirements. Minor actinides to be transmuted could be added to the 
fuel. The power density in the core will be limited to less than $100 \mathrm{MW} / \mathrm{m}^{3}$ for safety reasons. The fuel considered is made by $(\mathrm{U}, \mathrm{Pu}) \mathrm{C}$ or $(\mathrm{U}, \mathrm{Pu}) \mathrm{N}$ pellets packed between two plates of $\mathrm{SiC}$, which requires an extensive $\mathrm{R} \& \mathrm{D}$ to demonstrate its feasibility and validity.

Among the running GFR projects, we signal the materials testing reactor ALLEGRO - pursued by the Visegrád Group (CZ, HU, PL, SK) through the V4G4 Centre of Excellence and the French CEA with Euratom support - and two $2400 \mathrm{MW}$ reactors, one with indirect cycle and $(\mathrm{U}, \mathrm{Pu}) \mathrm{C}$ fuel, the other with a direct cycle and $(\mathrm{U}, \mathrm{Pu}) \mathrm{N}$ fuel. In the U.S., General Atomics is developing the Energy Multiplier Module (EM ${ }^{2}$, a $265 \mathrm{MW}_{e}$ small modular reactor employing a convert and burn core design allowing a 30-year continuous operation [15]. If fission products are periodically removed, $\mathrm{EM}^{2}$ can recycle its used fuel, producing one-fifth of the waste of current LWRs for the same amount of electricity. A peculiarity of $\mathrm{EM}^{2}$ is that it will be fabricated in a factory and transported by road to the site of operation.

24. Supercritical-Water-Cooled Reactor $(S C W R)$. - This thermal reactor concept is under consideration in the U.S. for the simplicity of its design [16]. It is an evolution of the super-heated steam cooled reactors operating at subcritical-pressure that were tested in the 1950s in both the U.S. and the USSR. Water heated above $647 \mathrm{~K}$, at a pressure higher than 22.07 MPa, becomes a supercritical fluid without boiling. In this state, the fluid has thermofluido-dynamical properties similar to those of saturated steam, with excellent heat transfer characteristics. The system can, therefore, afford a high power density and a compact geometry (the targeted capacity is $1700 \mathrm{MW}_{e}$ ). Steam generators are not needed, as the coolant, acting also as a moderator, can be used directly in the turbine for electricity generation. The absence of boiling eliminates instabilities related to the reactivity's void coefficient. With an outlet coolant temperature of $833 \mathrm{~K}$, a thermodynamic efficiency $\eta \sim 45 \%$ can be achieved in a supercritical Rankine cycle (fig. 6).

SCWRs would run on MOX fuel adopting a close cycle strategy. With a tight fuel rod lattice, a fast reactor core is possible. In both cases, a heavy-water SCWR could breed fuel (producing fissile U-233) from thorium-232 by neutron capture in the thermal region of the energy spectrum.

Engineering challenges arise from the high operating pressures and temperatures, producing strong mechanical and thermal stresses on vessel materials. The operating conditions require using high standard structural materials, and the manufacturing of the huge pressure vessel (about $5.3 \mathrm{~m}$ inner diameter and $46 \mathrm{~cm}$ thickness) would represent a difficult industrial endeavour.

Research and development activities are currently focused on the chemistry of supercritical water under neutron irradiation and materials properties related to microstructural stability, embrittlement, and creep resistance. Fuel cladding is also an issue for SCWRs in case of a loss of coolant accident, because the low water inventory would allow for transient temperatures not compatible with the conventional metallic cladding used in current LWRs. 


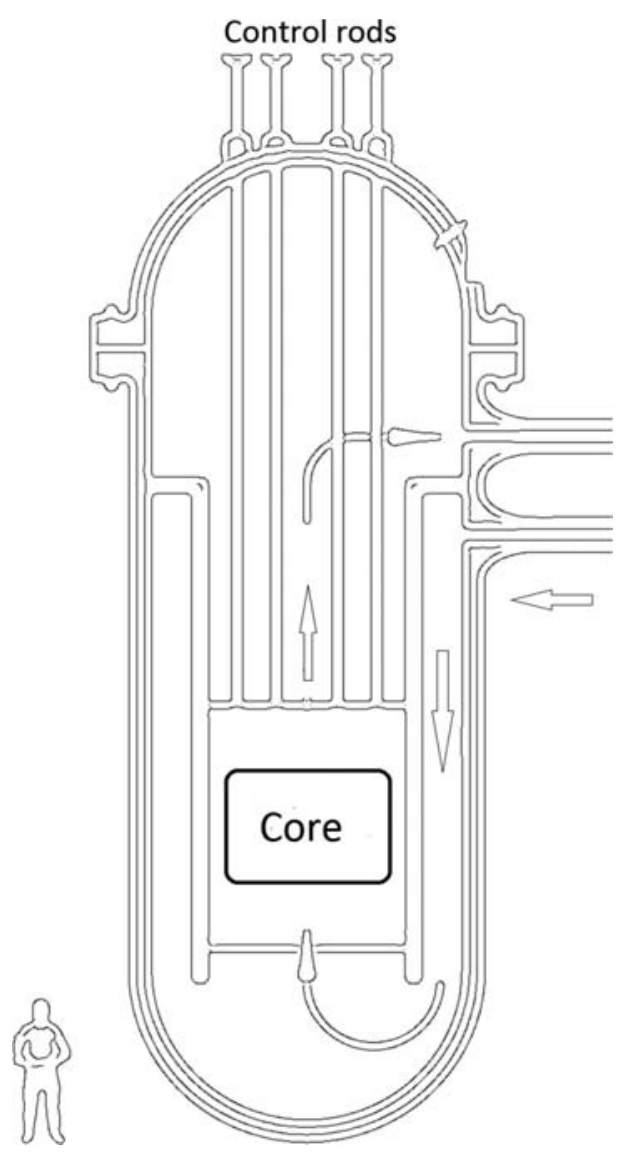

Fig. 6. - Schematic representation of a Supercritical-Water-Cooled Reactor.

2·5. Molten Salt Reactor $(M S R)$. - They were conceived in the late 1940 s and first developed by the U.S. Air Force in the 1950s, although MSR have never been used to power aircrafts. Between 1965 and 1969, an interesting test was run at the Oak Ridge National Laboratory, where the $8 \mathrm{MW}$ Molten Salt Reactor Experiment (MSRE) was maintained into operation for 20000 hours. A MSR can be a fast or a thermal reactor, depending on the core configuration. The latter makes use of a graphite moderator and is proliferation-resistant thanks to a low fissile material inventory.

The primary coolant is a mixed $(\mathrm{Li}, \mathrm{Na}, \mathrm{Zr}, \mathrm{Th}, \mathrm{U}$, and $\mathrm{Pu}$ ) fluoride or chloride salt in the liquid state at around $923 \mathrm{~K}$. The fuel (U, Th, or $\mathrm{Pu}$ ) can be either in solid rods, as foreseen in the Fluoride Salt High-Temperature Reactor (FHR), or dissolved directly into the molten salt. This latter option considerably simplifies the fuel fabrication. The liquid fuel molten salt reactor (MSR) is usually combined with a chemical reprocessing plant to remove fission products and recycle fissile materials as U-233 generated when a Th fuel cycle is implemented. In both type of molten salt reactors, the outlet coolant 


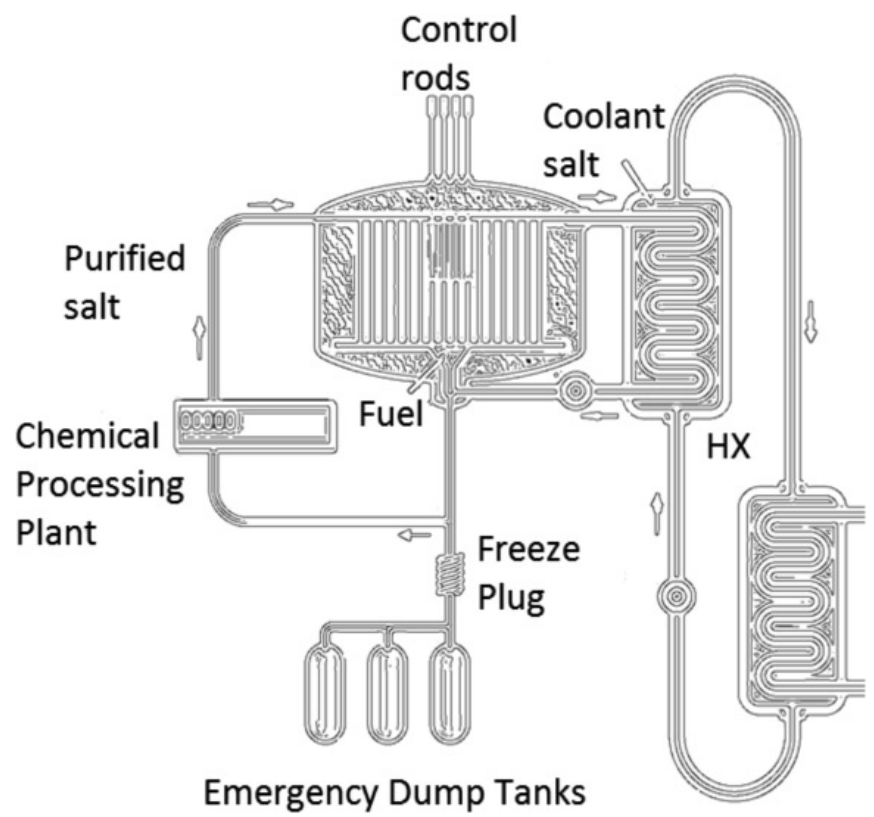

Fig. 7. - Schematic representation of molten salts reactor.

temperature is in the range $973-1073 \mathrm{~K}$ and the operating pressure is low (typically $<0.5 \mathrm{MPa}$ ). From the neutronics point of view, an advantage of MSR lies in the negative reactivity feedback coefficients in case of thermal expansion (the chain reaction slows down if the system is overheated). Safety passive methods are easily implemented and emptying the vessel is sufficient to shut down the reactor. This occurs without external interventions thanks to a plug at the bottom of the reactor vessel that melts when the temperature increases anomalously (fig. 7). Among current development projects we cite the TerraPower MCFR, mainly conceived for process heat and thermal storage, and the Elysium Molten Chloride Salt Fast Reactor (MCSFR) that is assumed to have a flexible thermal capacity, ranging from 125 to $2500 \mathrm{MW}$, and to consume spent nuclear fuel and weapons waste. The choice of chloride salts over fluorides avoids the use of Li-7 (eliminating concerns due to the management of tritium emissions), and the proliferation concerns associated with Li-6 (used as a source of tritium by fission and, directly, in singleand double-stage thermonuclear and boosted fission weapons).

There are, of course, several challenges for the development of MSR. Molten halide salts are corrosive and the high neutron flux, combined with the high operating temperatures, imposes severe conditions on structural materials that must withstand radiation damage and corrosion (the latter can in part be controlled via the electrochemical potential of the fuel, in practice by the $\mathrm{UF}_{3} / \mathrm{UF}_{4}$ ratio). The water-molten salts thermodynamic interactions are still poorly known and must be further investigated to assess safety-related scenarios, as in case of heat exchanger tube break. Controlling the chain 
reaction cannot be done by using control bars, as in the other nuclear reactor systems, and no poisons are foreseen: the emergency shut down (scram) must be made by emptying the core into dump tanks with appropriate geometries to avoid criticality. The reactivity modelling is complicated by the fact that delayed and prompt neutrons are generated at different positions, because of the moving fuel. Finally, the confinement of fission gases requires special attention.

2*6. Very-High-Temperature Reactor (VHTR). - These are systems conceived to be primarily dedicated to the cogeneration of electricity and hydrogen by thermochemical processes (fig. 8). The coolant is He gas, as for GFR. In a VHTR, however, a graphite moderator is used to thermalize the neutron energy spectrum. Because the operating temperature is too high for conventional fuel with metallic cladding (the outlet coolant temperature being $1273 \mathrm{~K}$ ), VHTR will burn fuel (U-Pu, Pu, MOX, U-Th, with 15\% enriched uranium) in the form of so-called tristructural isotropic (TRISO) particles. A TRISO particle is a small diameter $(0.6 \mathrm{~mm})$ fuel kernel, coated by successive layers of porous carbon, pyrolytic graphite, and silicon carbide. Such a coating is able to resist without failing even to the extreme temperature excursion that would occur in the case of a loss-of-coolant accident. Thousand of TRISO particles are packed inside a pebble, which is a graphite spherical shell with a diameter of a few centimetres. A bed of pebbles forms the core (with about $40 \%$ of the volume occupied by them). As graphite as a moderator material is less efficient than water, the core size is usually relatively big. To facilitate residual heat removal in off-normal conditions (loss of forced convection), the core has a annular geometry with inner and outer graphite reflectors that contain also control elements. The reactor refuels by adding fresh pebbles daily in at the top, as older ones are discharged from the bottom. Each pebble remains in the core for $\sim 3$ years and is circulated through the core up to six times to achieve full burn-up. In an alternative core design, the coated particles (TRISO) are embedded in prismatic graphite blocks through which the gas coolant flows. Such a configuration is pursued in U.S.A. and Japan.

A VHTR is designed to have high thermal efficiency in a direct cycle electricity production, with helium gas turbine directly set in the primary coolant loop. As the power density in the core is low $\left(\sim 10 \mathrm{MW} / \mathrm{m}^{3}\right)$, natural conduction can remove the decay heat. In the case of a modular reactor design, the power system will consist of several units, each one producing several tens of megawatts of electric power. The presence of graphite in the core raises a moderate risk of graphite fire if air enters into the system (a similar event occurred during the Windscale and Chernobyl accidents). Besides, water entering the core would produce changes in reactivity and production of inflammable gas. VHTR projects at an advanced stage include the X-Energy 100 Pebble-Bed Reactor (200 MW), whose conceptual design should be completed by the end of 2019, AREVA HTGR, and the High-Temperature Test Reactor, a $30 \mathrm{MW}$ system under development at Oarai, Japan. The Chinese $250 \mathrm{MW}$ pebble-bed HTR-PM is expected to start generation of electricity at the end of 2021. If this deadline is respected, it will be the first GenIV system to become operational. 


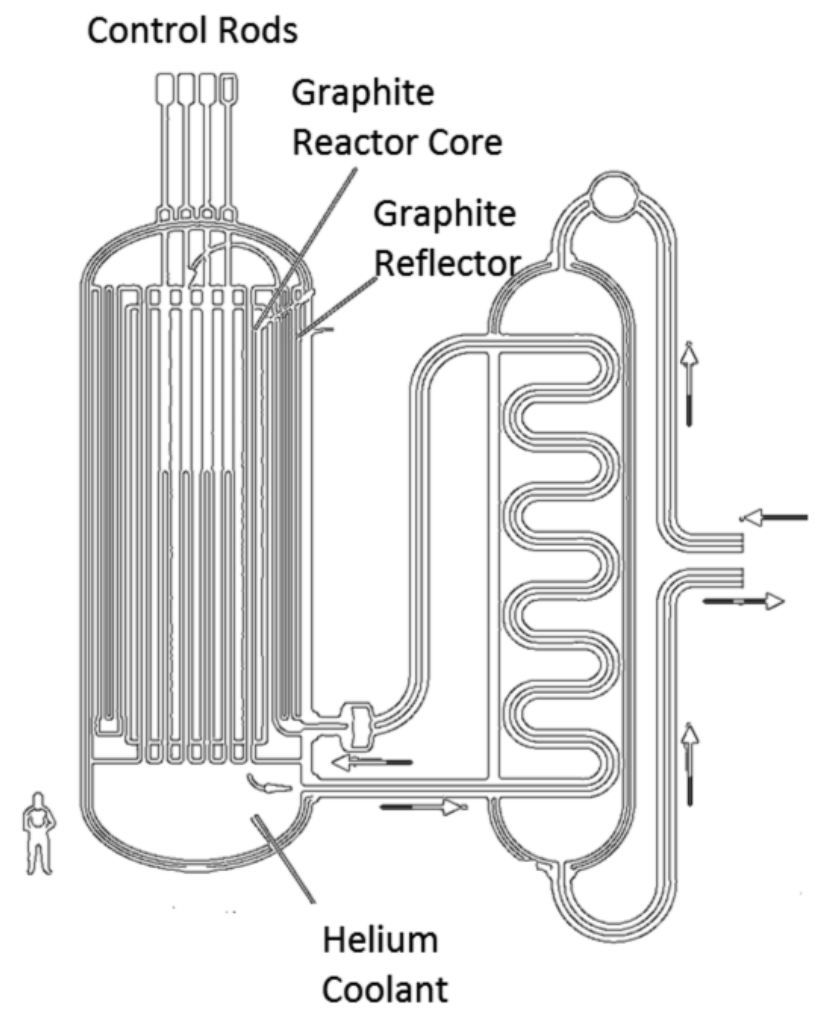

Fig. 8. - Schematic representation of a Very-High-Temperature Reactor.

\section{3. - Conclusions}

Updated versions of several nuclear technologies proposed and tested at the dawn of the nuclear era may prove viable for satisfying the increasing energy need of the planet population while mitigating the risks of disruptive climatic changes. However, the time scale to commercial deployment of these technologies will be measured in decades, while a fast expansion of low-carbon electricity supplies is needed immediately to achieve deep decarbonization goals. Generation-IV-based scenarios to mitigate global warming are discussed in $[17,18]$.

In the European Union, nuclear electricity ( $~ 25 \%$ of the 3.1 PWh total production) avoids the emission of 1000-1200 Mtonnes of $\mathrm{CO}_{2}$ /year, about twice the amount currently saved by renewable energy sources. It is, therefore, a key component of a mix of affordable energy sources for achieving the European Unions goals of GHG reduction by 2030. Moreover, the use of nuclear energy for district heating and industrial processes can further contribute to the reduction of GHG and is currently under investigation.

Adequate basic and applied R\&D programs are still needed to optimize and improve the safety and efficiency of existing, evolutionary and advanced concepts. Permanent 
solutions to spent-fuel and high-level nuclear waste management and disposal (possibly harmonized at continental scale) are urgently needed. New systems, like e.g. MYRRHA in Belgium, must be accompanied by the development of innovative safeguards. Proliferation control and nuclear forensic capabilities must be adapted to evolving scenarios. State of the art knowledge and scientific infrastructure for nuclear R\&D must be maintained. Transformational long-term applied and basic research must be deployed now to prepare the future.

We are grateful to Dr. V. V. Rondinella, Prof. R. Konings, and Prof. T. Fanghänel for their suggestions and their critical reading of the manuscript.

\section{REFERENCES}

[1] International Energy Agency, https://webstore.iea.org/global-energy-co2-statusreport-2018 (2019)

[2] Population Division of the Department of Economic and Social Affairs of the United Nations Secretariat, https://population.un.org/wpp/ (2019).

[3] Dones R., Heck T. and Hirschberg, S., Greenhouse Gas Emissions From Energy Systems: Comparison and Overview, Paul Scherrer Institut Scientific Report, 2003, Vol. IV: Nuclear Energy and Safety, edited by Smith B. and Gschwend B. (Villigen PSI, Switzerland) 2004, pp. 27-40.

[4] Eurostat, https://ec.europa.eu/eurostat/cache/infographs/energy/bloc-2a.html (2016).

[5] electricityMap API, https://www.electricitymap.org (2019).

[6] IAEA Power Reactor Information System, www.iaea.org/pris (2019).

[7] GIF R\&D Outlook for Generation IV Nuclear Energy Systems: 2018 Update, www.gen-4.org/gif/jcms/c_108744/7411-gif-r-and-d-outlook-update-web (2019).

[8] Briggs M. H., Gibson M. A. and Sanzi J., Electrically Heated Testing of the Kilowatt Reactor Using Stirling TechnologY (KRUSTY) Experiment Using a Depleted Uranium Core, https://ntrs.nasa.gov/archive/nasa/casi.ntrs.nasa.gov/20170007986.pdf (2019).

[9] Michal R., Nucl. News, 11 (2001) 128.

[10] Fink J. K. and Leibowitz L., Thermodinamic and Transport Properties of Sodium Liquid and Vapor, Argonne National Laboratory Report ANL/RE-95/2, https://www.ne.anl.gov/eda/ANL-RE-95-2.pdf (1995).

[11] Dalle Donne M. and Goetzmann C. A., "Gas-cooled fast breeder reactor design, development, and safety features", in Proceedings of the ANS Topical Meeting on GasCooled Reactors: HTGR and GCFBR (American Nuclear Society, Gatlinburg, USA) 1974.

[12] Waltar A. E. and Reynolds A. B., Fast Breeder Reactors (Pergamon Press, New York) 1981.

[13] Van Rooijen W. F. G., "Gas-Cooled Fast Reactor: A Historical Overview and Future Outlook", Sci. Technol. Nucl. Install., 2009 (2009) 965757.

[14] Simon R. H., Dee J. B. and Morita W. I., "Gas-cooled fast breeder reactor demonstration plant", Proceedings of the ANS Topical Meeting on Gas-Cooled Reactors: HTGR and GCFBR (American Nuclear Society, Gatlinburg, USA) 1974, p. 336. 
[15] General Atomics U.S.A., Advances in Small Modular Reactor Technology Developments (International Atomic Energy Agency, Vienna, Austria) 2018, pp. 183-186.

[16] OKa Y. and Koshizuka S., "Supercritical-pressure, Once-through Cycle Light Water Cooled Reactor Concept", Nucl. Sci. Technol., 38 (2001) 1081.

[17] Berger A. et al., "How much can nuclear energy do about global warming?", Int. J. Glob. Energy Issues, 40 (2017) 43.

[18] Berger A. et al., "Nuclear energy and bio energy carbon capture and storage, keys for obtaining $1.5^{\circ} \mathrm{C}$ mean surface temperature limit", Int. J. Glob. Energy Issues, 40 (2017) 240. 